\title{
Anti-inflammatory Activity of $N$-Naphthoyl D-Alanine in vivo
}

\author{
Michimasa Goto, ${ }^{-}$Himmi Kataoka, ${ }^{\dagger}$ Yoshie Araya, Masashi Kawasaki, ${ }^{\ddagger}$ Kin-ichi Oyama, ${ }^{\S}$ Masanori Semma, \\ Yoshio Ito, and Atsushi Ichikawa ${ }^{\dagger}$
}

\author{
Department of Chemical and Biochemical Engineering, Tovama National College of Technologv, \\ Hongo 13, Tovama 939-8630, Japan. 'E-mail: gotoGtovama-nctac.jp \\ ${ }^{\dagger}$ Department of Pharmacology, Factly of Pharmaceutical Sciences, Aukogata Homen's Lniversitv, \\ Kvabam-Cho 11-68, Koshien, Nishinomiva cin, Hvogo Prefecture 663-8179. Japan \\ -Faculty of Engineering. Tovama Prefectural Lniversit, 5180 Kurokata, Kosugi-Hachi, Tovama 939-0398, Japan \\ ¿Chemical Instrument Room, Research Center for Materials Science, Nagova Universin. Chikusa, Nagova 464-01, Japan \\ Received December 3, 2008, Accepted February 25, 2009
}

Key Words: D-Alanine. Anti-inflammatory activity. Zigzag structure, Carrageenan, COXs

Ibuprofen and naproxen are widely used for the clinical treatment of inflammatory diseases and are classified as propionic acid type anti-inflammatory drugs. Such acid type anti-inflammatory drugs exert their effect by inhibiting the cataly tic activity ${ }^{1,2}$ of cyclooxygenases (COXs) ${ }^{3}$ that catalyze the biosy nthesis of prostaglandins from arachidonic acid. For expression of COXs-inhibitory activity, such compounds requir 1) a carboxylic function ${ }^{4} 2$ ) $S$-configuration at the chiral center. ${ }^{+}$and 3 ) a zigzag structure ${ }^{5}$ drawn in bold lines as shown in Fig. 1.

We attempted to have $N$-naphthoyl D-alanine $(R-1)^{6}$ ? expressing anti-inflammatory activity in a COXs-inhibiting manner by reproducing the zigzag stnicture of naproxen in $R-1$ as shown in Fig. 1. The reasons for expecting such anti-inflammatory activity are as follows: 1) the stereochemistry at the chiral center (C3) was the same as that of $\mathrm{D}$-alanine 2) the planarity between $\mathrm{C} 4-\mathrm{C} 5$ in naproven might be replaced with the double bond nature of the amide function (N--C5) 3) the zigzag structure in naprosen could be mimicked as the zigzag stnicture drawn in bold lines in Fig. 1 4) the planarity between $\mathrm{C} 5-\mathrm{C} 6$ in $R-1$ might be obtained by the $\pi$ electron donation from the aromatic ring to the amide carbonyl function 5) $R-1$ had a naphthalene ring that might settle on the planar domain of the binding site of the COXs advocated by Gund $e t a I^{8}$

First, anti-inflammatory activities against four compounds. $R-1, S-1,2$ and $R-3$, were evaluated by carrageenan-induced mouse foot-pad swelling assay ${ }^{10.11}$ at a dose of $150 \mathrm{mg} / \mathrm{kg}$ and statistical analyses were performed by Dunnett's method. Obtained data on the percentage inhibition and judgments on significant differences from the control group are summarized in Table 1.

$R-1$ reduced carrageenan-induced mouse foot-pad swelling by $45.68 \pm 9.87 \%$ and this reduction was judged to be a

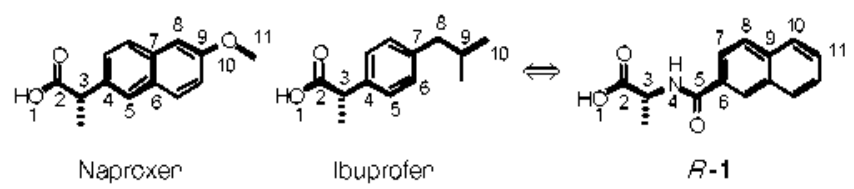

Figure 1. Zigzag structure in naproxen, ibuprofen and $R-1$. significant difference (judged as " $\$$ "), but $S-1$ did not exhibit anti-inflanmatory activity, that is. compound $S$-1 showed no significant difference (judged as "NS"). These two results are consistent with the structure-activity relationship of propionic acid type anti-inflammatory drugs. Compound 2 , which had no methyl function at the 3rd position. showed no inhibitory effect on carrageenan-induced mouse foot-pad swelling. This meant that the down methyl group at the 3rd position was necessary to exert the reduction effect on carrageenan-induced mouse foot-pad swelling.

In order to determine whether the planarity between N4-C5 by the double bond nature of the amide function was important for the activity. the inhibitory activity of compound $R-\mathbf{3}$ was examined. The features of $R \cdot \mathbf{3}$ were to have the same stereochemistry as $R-1$ and a poorer double bond nature between N4-C5 than that of $R-1$ because of having a thioamide function instead of an amide one. This assay revealed that $R-3$ had no effect on carrageenan-induced mouse foot-pad swelling. This result indicates a significant planarity between N4-C5 for exertion of the inhibitory activity against carrageenan-induced mouse foot-pad swelling. In addition the offset of the inflammatory activity of $R-3$ might be partially due to the absence of planarity between

Table 1. Results of inhibition assay of carrageenan-induced mouse foot-pad swelling

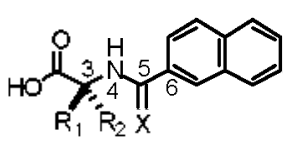

$1-3$

\begin{tabular}{cccccc}
\hline Compound & $\mathrm{R}_{1}$ & $\mathrm{R}_{2}$ & $\mathrm{X}$ & \% of inhibition & $\mathrm{S}^{\circ}$ or NS \\
\hline$R-1$ & $\mathrm{H}$ & $\mathrm{CH}_{3}$ & $\mathrm{O}$ & $45.68 \pm 9.87$ & $\mathrm{~S}$ \\
$S-1$ & $\mathrm{CH}_{3}$ & $\mathrm{H}$ & $\mathrm{O}$ & $1.8 \pm 7.99$ & $\mathrm{NS}$ \\
2 & $\mathrm{H}$ & $\mathrm{H}$ & $\mathrm{O}$ & $15.43 \pm 7.35$ & $\mathrm{NS}$ \\
$R-3$ & $\mathrm{H}$ & $\mathrm{CH}_{3}$ & $\mathrm{~S}$ & $9.68 \pm 6.67$ & $\mathrm{NS}$ \\
\hline
\end{tabular}

"Assays were carried out at a dose of $150 \mathrm{mgkg}$ (ref. 11 ). Cf. Percentage inhibition of naproxen: $40.68 \pm 4.4(30 \mathrm{mg} \mathrm{kg}$. p.o.). Each value represents the mean $=$ S.E. for $5-7$ mice. ${ }^{b} S$ means to differ significantly and NS means no significant difference from the control group (statistically analyzed by Dunnett's method. $P<0.05$ ). 
Table 2. Results of inlhibition assay of carragenan-induced mouse foot-pad swelling and calc. $\log P$ values of aromatic ning moiety
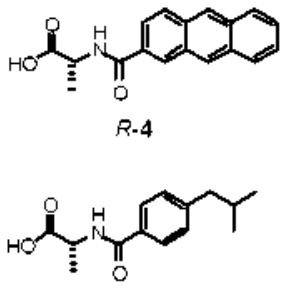

R-7
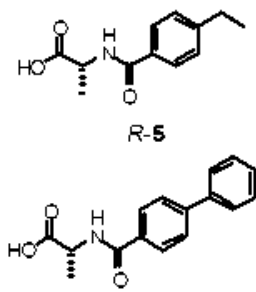

R-8
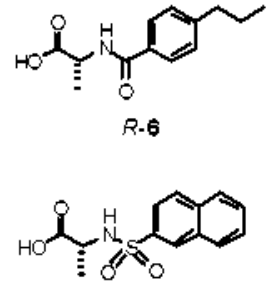

R.9
Compound \%ofinhibition ${ }^{a}$ Sor $\mathrm{NS}^{b} \quad$ Calc. $\log P$ value ${ }^{c}$ of aromatic ring moiets.

\begin{tabular}{lcll}
\hline$R-4$ & $0.10 \pm 6.34$ & NS & 4.03 \\
$R-5$ & $25.44 \pm 14.12$ & NS & 2.94 \\
$R-6$ & $-1.4 \pm 5.1$ & NS & 3.36 \\
$R-7$ & $9.20 \pm 3.8$ & NS & 3.69 \\
$R-8$ & $36.97 \pm 8.59^{4}$ & $\mathrm{~S}^{*}$ & 3.71 \\
$R-9$ & $31.69 \pm 8.94$ & $\mathrm{~S}$ & 3.03 \\
\hline
\end{tabular}

Assays were carried out at a dose of $150 \mathrm{mg}$ kg (ref. 11 ). $\mathrm{Cf}$. Percentage inhibition of naproxen: $40.68=4.4(30 \mathrm{mg} k \mathrm{~kg}$, p.o.). Each value represents the mean $=S$. E. for $5-7$ mice. ${ }^{t} S$ means to differ significantly and NS means no significant difference from the control group (statistically analyzed by Dumett's metlod. $P .0 .05$ ). Asterisk indicates signiticant difference $(\dot{P}<0.01)$. 'See. ref. 12 . "This datum was obtained at a dose of $300 \mathrm{mg} \mathrm{kg}$.

C5-C6 by changing the amide moiety to thioamide function.

All the results obtained from the assays using $R-1, S-1,2$. and $R-3$ were thought to support the exertion of anti-inflammatory activity by $R-1$ being attributed to the inhibition of COXs activity. although indirectly (not in vitro assay).

Next. we performed assays on the anti-inflammatory effect of amides constnicted by $\mathrm{D}$-alanine and aromatic acids other than naphthoic acid. We evaluated six compounds $(R-t-R-9)^{9}$ for anti-inflammatory activities by carrageenan-induced mouse foot-pad swelling assay ${ }^{\text {ji.11 }}$ with a usual dose of 150 $\mathrm{mg} / \mathrm{kg}$. The percentage inhibition. determinations of S or NS, and $\log P$ values ${ }^{1}{ }^{2}$ of aromatic ring moiety are listed in Table 2 . Since the hydrophobicity of the active site of the enzyme is generally recognized to be high, a highly hydrophobic compound might have an advantage as a candidate $\mathrm{COXs}$ inhibitor binding to the active site of COXs.

Compound $R-4$, which had considerably high hydrophobicity $(\log P$ value of anthracene moiety: 4.03 ). did not have the potential to reduce carrageenan-induced mouse foot-pad swelling. This invalidity was attributed to the antluracene ring of $R-4$ being too large compared with the size of the planar binding domain in the active site of COXs. Compound $R-7$ had the same aromatic ring moiety as ibuprofen. and compound $R-5$ and $R-6$ had ones whose side chain length on the benzene ring was shorter than the side chain length of $R-7$. Compounds $R-5-R-7$ were expected to have anti-inflammatory activities because of the same or greater than $\log P$ values (2.94 for $R-5,3.36$ for $R-6$. and 3.69 for $R-7$, respectively) compared with $R-1$ (3.03). However. none of them had any inflammatory activity. These results indicate the importance of there being no side chains on the benzene ring but rather the size of the aromatic ring for exertion of anti-inflammatory effect. Reproducibility of $S$ or NS could not be obtained for compound $R-8$ with the usual dose of $150 \mathrm{mg} / \mathrm{kg}$. so assay at a dose of $300 \mathrm{mg} / \mathrm{kg}$ was performed. In this case. clear anti-inflammatory activity was observed reproducibly. athough it was weak (inhibition by $36.97 \pm 8.59 \%$ ).

Finally. anti-inflanmatory activity against compound $R-9 ., 13$ which had sulfonamide moiety instead of an anuide part. was investigated. This $R-9$ was predicted not to have the activity since the polarity of the sulfone function was too high to bind to the hydrophobic binding site of the COXs. Unexpectedly. this conpound exhibited a significant inhibition effect on carrageenan-induced mouse foot-pad swelling by $31.69 \pm 8.94 \%$.

In conclusion, carrageenan-induced nouse foot-pad swelling assay revealed the significant potential of anti-inflammation of $N$-naphthoyl D-alanine $(R-1)$. This anti-inflammatory activity was attributed to COXs inhibition because of no activity by $N$-naphthoyl L-alanine $(S-1)$. As aromatic ring moiety was adopted, not a benzene ring having an alkyl chain. but a naphthalene ring was suitable for exertion of antiinflanmatory activity. The zigzag structure, especially the planarity between N4-C5 derived from the double bond nature of the amide function, was important.

\section{Refeiences}

1. Varle, J. R. Nature New Biol. 1971, 231, 232.

2. Black, R. C. Trens Phamacol. Sci. 1981, 2, 310.

3. Halzman, M. T.; Turk, J.; Shomick, L. P. J. Biol. Chent. 1992, 267,21438

4. Yamada, K: Kanaoka, Y.: Iwasaki, Y. Medicinal Chemistn th ed: Koudanshya Scientific Co: Tokyo, 1998; p 171

5. Appleton, R. A.; Brown, K. Prostaglandines 1979, 18, 29.

6. (a) Allenmark, S.; Bomgren, B.; Boren, H. J. Chromatography 1983, 26t, 63. (b) Miura, M: Terashita, Y: Tanaka, M. Chomtatography 1998, 19,74.

7. DeRuiter, I.: Davis, R. A.: Wandrekar, V. G.: Mayfield, C. A. J. Hed. Chent $1991,34,2120$.

8. Gund, P.: Shen, T. Y.: Sharp and Dohme, M. J. hed. Chem. $1977,20,1146$

9. All compounds except for $R-3$ were obtained by hydrogenoly sis of conesponding benzyl esters with $10 \% \mathrm{Pd} / \mathrm{C}$ in THF after condensing conesponding aromatic acid chlorides and alanine benzyl ester or glycine benzyl ester in $\mathrm{CHCl}_{3}$. Optical purity of all synthesized $\mathrm{X}$-acylated D- and L-alanine benzyl esters was guaranteed by HPLC analysis through chiral cell-OB-H (DAICEL Co., Japan) as over $98 \%$ ee. Optical purity of $R-3$ was determined as $66 \%$ ee

10. Expenimental Hontols for Bio-phomacettical Sciences 12 , Inflammation and Allergy, 1-1.; Ohuchi K., Ed.; Hirokawa Co: Tokyo, 1993: p 18

11. Kataoka, H.; Horiyama, S.; Yamaki, M.; Oku, H.; Ishiguro, K.; Katagi, T.: Takayama, M.; Semma, M.: Ito, Y. Biol Pham. Bull. 2002, 25, 1436 .

12. All $\log P$ values were obtained by calculating with ChemDraw Ultra Software ver.10.0.

13. (a) Miura, M:; Funazo, K.; Tanaká, M. Anal Chimica Acta 1997, 357, 177. (b) Colles, Wm. M.; Gibson, C. S. J. Chem. Soc., Trans. 1924. 125.2505. 